\title{
Rotation, mass loss and nucleosynthesis
}

\section{Georges Meynet}

\author{
Geneva Observatory, University of Geneva, CH-1290 Sauverny \\ email: georges.meynet@obs.unige.ch
}

\begin{abstract}
We list below a few problems whose resolution might bring new lights on the impacts of the first stellar generations on the early phases of the evolution of the galaxies.
\end{abstract}

Keywords. Stars: evolution, rotation

For metallicities between those of the Small Magellanic Cloud and of the solar neighborhood, rotating models better reproduce the observed characteristics of stars than non-rotating models (see, e.g., Meynet et al. 2006). When the same physical processes as those necessary to obtain good fits at high metallicity are accounted for in metal poor stars, one notes that stars are strongly mixed and may lose large amounts of material. These features might be helpful for explaining puzzling observational facts, as the synthesis of important amounts of primary nitrogen by massive and intermediate mass stars (Ciappini et al. 2006), the existence of C-rich extremely metal poor stars (Meynet et al. 2006), the chemical inhomogeneities observed in globular clusters (Decressin et al. 2007), the presence of very He-rich stars in $\omega$ Cen (Maeder \& Meynet 2006).

To go ahead in these areas of research, we need to answer the following questions:

(a) How the initial distribution of the rotational velocities vary with the metallicity?

(b) What is the main physical mechanism responsible for determining the rotation of massive stars on the ZAMS?

(c) For a given initial mass star, initial rotation velocity, at a given age, the surface enrichments are higher at lower $Z$. Is this prediction confirmed by the observations?

$(d)$ How the surface velocity vary at the surface of stars of different initial masses and metallicities along the Main-Sequence phase?

(e) What are the conditions for the surface velocity of a star to reach the critical velocity? (N.B. The critical velocity is the equatorial rotation velocity such that the centrifugal acceleration exactly compensates for the gravity.) When does it happen? What happens at the critical limit?

(f) What happens for what concerns mass loss, when important amounts of CNO elements appear at the surface as a consequence of rotational mixing?

$(g)$ What is the role of the magnetic field and of the gravity waves in stellar interiors? The list is not exhaustive, but these few questions already represent serious challenges for the years to come.

\section{References}

Decressin, T., Meynet, G., Charbonnel, C., Prantzos, N., \& Ekström, S. 2007, A\&\&A, 464, 1029 Chiappini, C. , Hirschi, R., Meynet, G., Ekström, S., Maeder, A., \& Matteucci, F. 2006, AשA (Letters), 449, L27

Maeder, A., \& Meynet, G. 2006, A\&A (Letters), 448, L37

Meynet, G., Ekström, S., \& Maeder, A. 2006, A\& A, 447, 623 